\title{
Molecular docking study of L-Asparaginase I from Vibrio campbellii in the treatment of acute lymphoblastic leukemia (ALL)
}

\author{
Abdul Khader Sultan Mohideen
}

\begin{abstract}
The potential use of asparaginases has gained tremendous significance in the treatment of acute lymphoblastic leukemia (ALL). Earlier studies suggest L-asparaginases (L-ASP) extracted from Escherichia coli and Erwinia aroideae regulates L-asparagine (L-Asn) from the circulating blood. Prolonged exposure to these enzymes may lead to hypersensitivity reactions. So, it is important to find novel asparaginases with anti-cancer properties. The three-dimensional structure of L-ASP I from Vibrio campbellii was determined by homology modeling using EasyModeller v.4.0. The structure was validated with quality indexing tools and was deposited in Protein Model DataBase. Molecular docking was performed between L-ASP I and ligand substrate L-Asn to study enzyme-substrate interactions. Qualitative and quantitative analysis of L-ASP I enzyme was found to be reliable and stable with a significant protein quality factor (LG score: 7.129). The enzyme is a dimer, belongs to $\alpha / \beta$ class of proteins. The active sites comprises of $\mathrm{N}$-glycosylation site and a catalytic triad (T14-S117-D92). The binding energy of the docked complex was calculated to be $-7.45 \mathrm{kcal} / \mathrm{mol}$. The amino acid T14 identified as a primary nucleophile essential for catalytic reaction. The enzyme L-ASP I of $V$. campbellii provides a detailed view of structure and functional aspects with ligand substrate L-Asn. This in silico investigation has explicitly demonstrated for the first time that cytosolic L-ASP Type I of V. campbellii to have a catalytic triad which was attributed only to periplasmic L-ASP Type II. Thus, L-ASP I can serve as anti-leukemic agent in the treatment, management and control of ALL.
\end{abstract}

Keywords: L-Asn, L-ASP I, In Silico, anti-leukemic agent

PG \& Research Department of Zoology, The New College (Autonomous), Affiliated to University of Madras, Tamilnadu, India

Corresponding author: S. A. K. Mohideen E-mail: smdbio@yahoo.co.in

DOI: $10.2478 /$ ebtj-2020-0002
(C) 2020 Authors. This work was licensed under the Creative Commons AttributionNonCommercial-NoDerivs 4.0 License.

\section{Introduction}

L-asparaginase (L-ASP) is a therapeutically significant enzyme, catalyzes the hydrolysis of L-asparagine (L-Asn) to produce aspartic acid and ammonia (Fig. 1). Bacterial type asparaginases are classified into subtypes I and II, which is defined by their intra or extracellular localization (1). L-ASP Type I (cytosolic) has a lower affinity for L-Asn, while type II (periplasmic) has a high substrate affinity. L-Asn is an essential amino acid synthesized from aspartate by asparagines synthetase in the presence of ATP and $\mathrm{Mg}++(2)$. L-Asn is indispensable to protein synthesis in some leukemic cells as they cannot produce this amino acid de novo due to absence/deficiency of the enzyme asparagine synthetase (3). Since certain tumours exhibit abnormally high requirements for asparagine, asparaginases are tested as antitumour agents (4).

The enzyme asparaginase is present in most of the microbes like Aerobacter, Bacillus, Pseudomonas, Serratia, Xanthomonas and Photobacterium (5). In addition, it is also found in Streptomyces, Vibrio and Aspergillus (6-8). In reality, not all L-ASP have anticancer properties as it is correlated with the affinity of the enzyme to the substrate (9). There is a plethora of studies pertaining to L-asparaginases extracted from Escherichia coli and Erwinia carotovora regarding anti-cancer or cytotoxic activity (10). The treatment of ALL has improved to a large extent for the past few decades due to the advancement of well-de- 

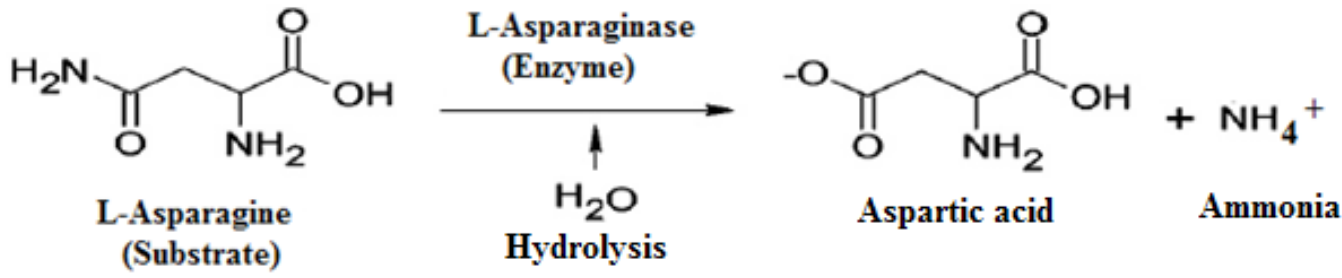

Figure 1. Schematic representation of the reaction mechanism between L-Asparaginase and L-asparagine. The enzyme catalyzes the hydrolysis of L-asparagine into aspartic acid and ammonia depriving the tumour cells to utilize this amino acid and inhibiting tumour growth.

signed protocols. While long-term event-free survival rate in children was found to be around $80 \%$ (11), compared to adults with 38-50\% long-term survivability (12).

Different tumour cells require L-Asn for the synthesis of proteins but they are deprived of this essential amino acid in the presence of L-ASP (13). Thus, cancer cells cannot grow in the absence or diminished expression of L-Asn. The inability to synthesize L-Asn by these tumour cells entirely depends on L-Asn from the circulating blood (14).

Therefore, depletion of L-Asn results in the death of leukaemic cells. A tumour inhibitory activity was observed only with asparaginases isolated from Escherichia coli, Erwinia aroideae and Serratia marcescens (15). The administration of these therapeutic enzymes for a long time exposure has resulted in anaphylactic shocks (16). Hence, the need for discovering novel serologically different L-ASP from new microbial strains having similar therapeutic effect and efficacy is the need of the hour (17).

Many research organizations have studied asparaginases production and purification in order to minimize impurities which may cause hypersensitivity or allergic reactions (18). The prolonged use of these enzymes may serve as antigens to the host body and elicit the production of antibodies in the tissues, which may interfere with the efficacy of the drug. Therefore, it is essential to search for novel L-ASP with medical properties. Several crystal structures of asparaginases from a wide variety of organisms have been determined earlier, (19). but still there is a paucity of structural information. Hence, the present study is focussed on modeling of L-ASP I enzyme from Vibrio campbellii to gain structural insights. Molecular docking is performed between L-ASP I and L-Asn to study the enzyme-substrate interactions and functional characterization of the enzyme by applying in silico methods to unravel its anti-cancer properties for therapeutic purposes.

\section{Materials and Methods}

\section{Sequence retrieval and analysis}

The amino acid sequence of asparaginase enzyme was searched using the UniProtKB at the ExPASy (http://www.uniprot.org/). The enzyme L-ASP I from Vibrio campbellii was selected with Acc. No. KGR34260.1 and the sequences were extracted in FASTA format for further analysis.

Physical and chemical parameters of the entire L-ASP I en- zyme was computed by ProtParam tool (http://web.expasy.org/ protparam/) which analyzed for molecular weight, theoretical pI, amino acid composition and grand average of hydropathicity (GRAVY). The antigenicity of the enzyme was predicted with AllerTOP v.2.0 (20). SOPMA server was used to predict the secondary structure of L-ASP I enzyme to assess the conformational information about positional possibilities of the $\alpha$-helices, $\beta$-strands, turns, random and coils within the protein structure (21).

\section{Homology modelling, structure validation and docking of L-ASP I enzyme}

BLASTp search of L-ASP I enzyme with default parameter was performed against the Brook Haven Protein Data Bank (PDB) to find the suitable template for comparative or homology modelling (http://blast.ncbi.nlm.nih.gov/). The PDB structures based on sequence coverage and identity were retrieved in PDB format and stored for further analysis. The theoretical structure of L-ASP I enzyme from $V$. campbellii was generated using EasyModeller v.4.0 (22). The software generated several preliminary models which were taken into consideration based on Discrete Optimization of Protein Energy (DOPE). The model with the lowest DOPE score was selected for model refinement and validation. The predicted structure was visualized using PyMOL molecular graphics viewer v.2.2.0 (www.pymol.org).

The predicted model was assessed by PROCHECK, Structural analysis and verification server (SAVES) (http://nihserver.mbi.ucla.edu/SAVES) which determines the stereochemical aspects along with the main chain and side chain parameters with comprehensive analysis (23).

The model quality was evaluated for different parameters like Root Mean Square Deviation (RMSD) using TM-score (https://zhanglab.ccmb.med.umich.edu/TM-score/), Errat for evaluating the progress of crystallographic model building and refinement, ProSA for predicting the Z-score and ProQ to assess the protein quality using a suitable template (24).

The functional assessment of the predicted model was carried out by searching against the PROSITE database and the active site residues of the modeled L-ASP I enzyme was predicted by Galaxy server (25).

L-Asn was extracted from the Library of 3-D Molecular Structures in PDB format (Fig. 2) (http://www.nyu.edu/pages/mathmol/library/life/life1.html /) to dock the catalytic site 


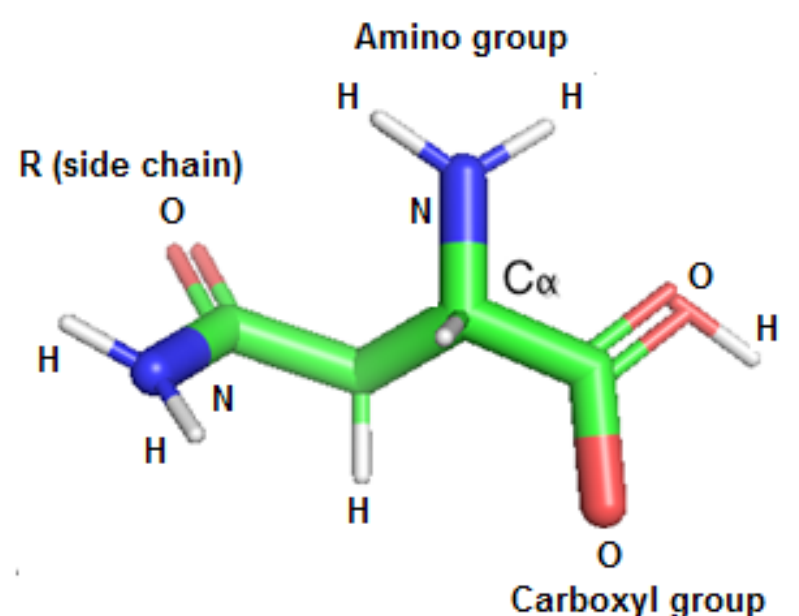

Figure 2. Crystal structure of L-asparagine (L-Asn) amino acid molecule. The L-Asn contains a central a-carbon atom to which both the amino (blue) and carboxyl groups (red) are attached. The other two bonds of the a-carbon atom are satisfied by the hydrogen (white) atom and the R group- side chain (Retrieved from Library of 3D Molecular Structures).

of L-ASP I using ArgusLab software v4.0 (26). In the docking process, the ligand substrate L-Asn and L-ASP I binding sites were prepared based on the results obtained from Galaxy server prior to molecular docking. An empirical scoring function (AScore) and docking engine (ArgusDock) were included in the ArgusLab program. The energy minimization was performed with universal force field molecular mechanics. The calculations were set to dock mode and flexible mode with grid resolution $0.40 \AA$. The pose with the least binding energy was calculated as the scoring function which exhibits the enzyme -ligand substrate interactions. The modes of binding between the docked complexes were visualized using PyMOL and LIGPLOT (27). The docking was further validated by uploading both the structures (L-Asn and L-ASP I) to PatchDock server (28).

\section{Results}

Physico-chemical parameters and amino acid composition of L-ASP I enzyme

The L- Asp I enzyme has a molecular weight of $36.9 \mathrm{kDa}$ with a theoretical isoelectric point (pI) 5.25. The Grand average of hydropathicity value for protein is -0.142 and the instability index (II) computed to be 43.21 . The enzyme is predicted as a non-allergen.

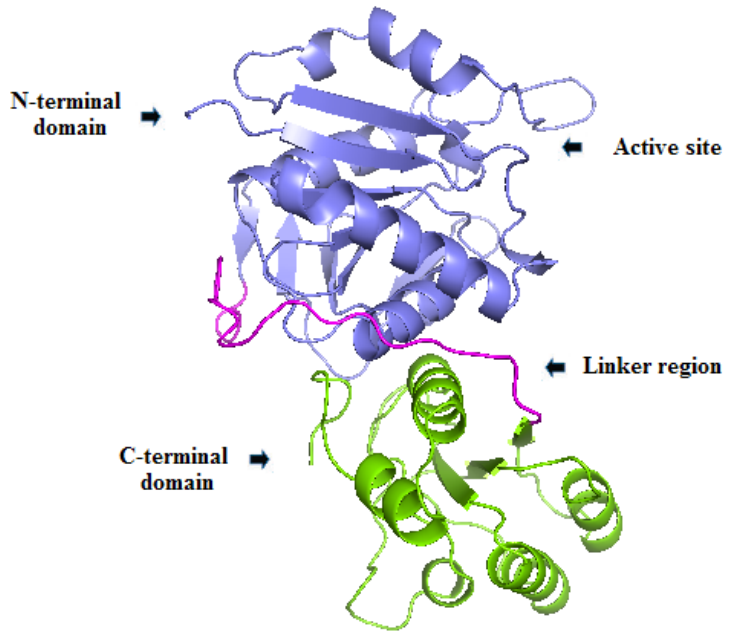

Figure 3. Modeled dimeric structure of L- Asparaginase I from $V$. campbellii. The two monomers (large and small) are shown in blue and green respectively. The larger and smaller monomers comprising of $\mathrm{N}$-terminal and $\mathrm{C}$-terminal regions.

The secondary structure prediction of the entire sequence of L-ASP I enzyme using SOPMA (with default parameters) showed that the protein having the composition of Helix = $35.61 \%$, Coil $=32.34 \%$, Strand $=20.77 \%$ and Beta-turn $=$ $11.28 \%$ respectively. It is clear from this secondary structure prediction that this enzyme belongs to $\alpha / \beta$ class of proteins.

The three- dimensional (3D) structure of L-ASP I enzyme was determined by homology modeling. BLASTp analysis (http://www.ncbi.nlm.nih.gov/) with the entire amino acid sequence of the L-ASP I enzyme of $V$. campbellii against PDB database showed good alignment with crystal structures of microbial origin, PDB ID: 2OCD_A, 2P2D_A, 2HIM_A and 3NTX_A (https://www.rcsb.org). The templates were selected based on sequence coverage and identity for modeling the enzyme (Table 1). The model with the lowest DOPE score (-38053.05) predicted by EasyModeller was considered for further analysis. The predicted 3D structure of the L-ASP I enzyme mainly composed of helices, coils, loops, strands and with traces of beta turns.

\section{Description of L-asparaginase I enzyme}

The modeled enzyme subunit contains two $\alpha / \beta$ domains connected by a linker region (Fig. 3 ). The dimer is characterized by alternating $\alpha$-helices/ $\beta$-sheets running along the backbone

Table 1. Blastp report of amino acid sequences producing significant alignments with L-ASP I of $V$. campbellii

\begin{tabular}{|c|c|c|c|c|c|}
\hline PDB ID & Query coverage (\%) & Max Identity (\%) & E value & Gap (\%) & Positive (\%) \\
\hline 2OCD_A & 100 & 85 & $8 \mathrm{e}-168$ & 0 & 91 \\
\hline 2P2D_A & 99 & 73 & $4 \mathrm{e}-139$ & 0 & 84 \\
\hline 2HIM_A & 99 & 73 & $2 \mathrm{e}-138$ & 0 & 84 \\
\hline 3NTX_A & 99 & 70 & $2 \mathrm{e}-132$ & 0 & 82 \\
\hline
\end{tabular}




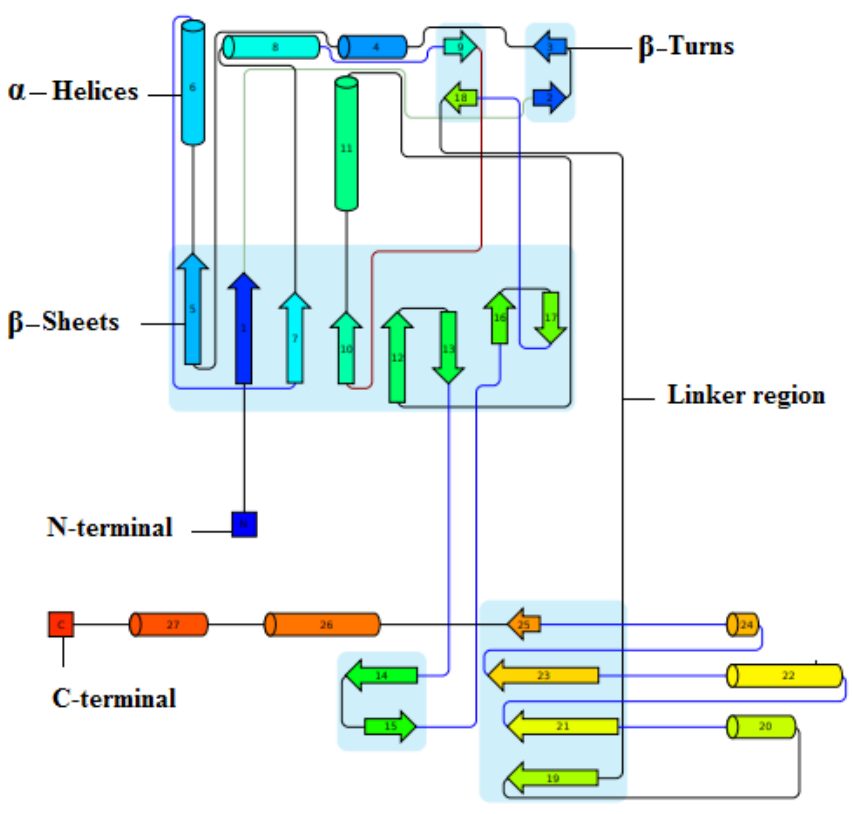

Figure 4. Topology of the L-Asparaginase I from V. campbellii with large arrows showing $\beta$-sheets, cylinders representing a-helices and small arrows indicating $\beta$-turns on both the $\mathrm{N}$-terminal and C-terminal regions of the dimeric enzyme. The two monomers are connected together by a linker region comprising of coil proteins (cartoon representation using Pro-origami).

of the N-terminal domain. The larger $\mathrm{N}$-terminal domain (residues 1-189) of the dimer contains four right-handed $\alpha$-helices with $\alpha-1$ and $a-4$ located on one side which forms the outer surface of the enzyme called accessible molecular surface or solvent exposed area. There are ten $\beta$-sheets; four of them are positioned in the interior from $\beta-1$ to $\beta-4$ which is arranged parallel, while $\beta-5$ leaves the main sheet to form a single small hairpin loop. The sheets from $\beta-5$ to $\beta-10$ are aligned antiparallel to each other. The helices $\alpha-2$ and $\alpha-3$ are found on the opposite side near the domain interface.

The linker sequence (magenta) connects the two monomers.

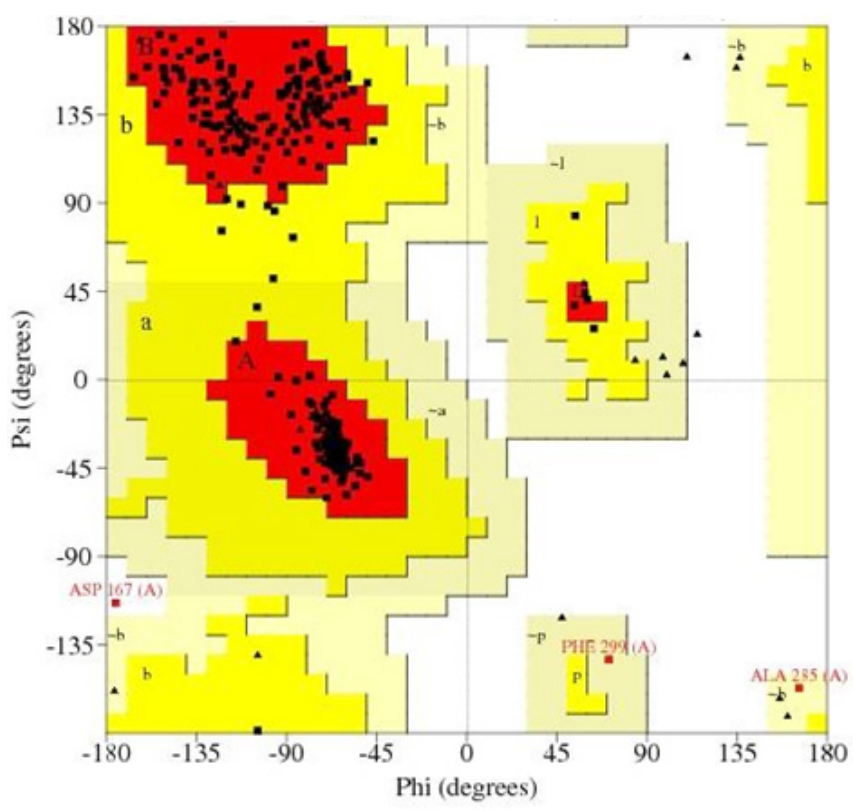

Figure 5. The Ramachandran map for L-Asparaginase I of $V$. campbellii shows the phi-psi torsion angles for all residues. The shading on the plot represents different regions. The red color corresponds to the core regions representing the most favourable combinations of phi-psi values.

The smaller C-terminal domain (residues 213-337) comprising of four right-handed $\alpha$-helices with $\alpha-1$ and $\alpha-2$ present within the distal region of the domain interface. There are three $\beta$-sheets occupying the interior region namely $\beta-1, \beta-2$ and $\beta-3$ which are arranged parallel while the helices $\alpha-3$ and a-4 are located on the inter-domain side of the enzyme. The linker sequence is made of coil protein which connects the two structural domains of the dimer from the amino acid residues 190-212. The topology of L-ASP I structure showing $\alpha$-helices, $\beta$ - sheets, turns and linker region is represented in a cartoon form (Fig. 4).
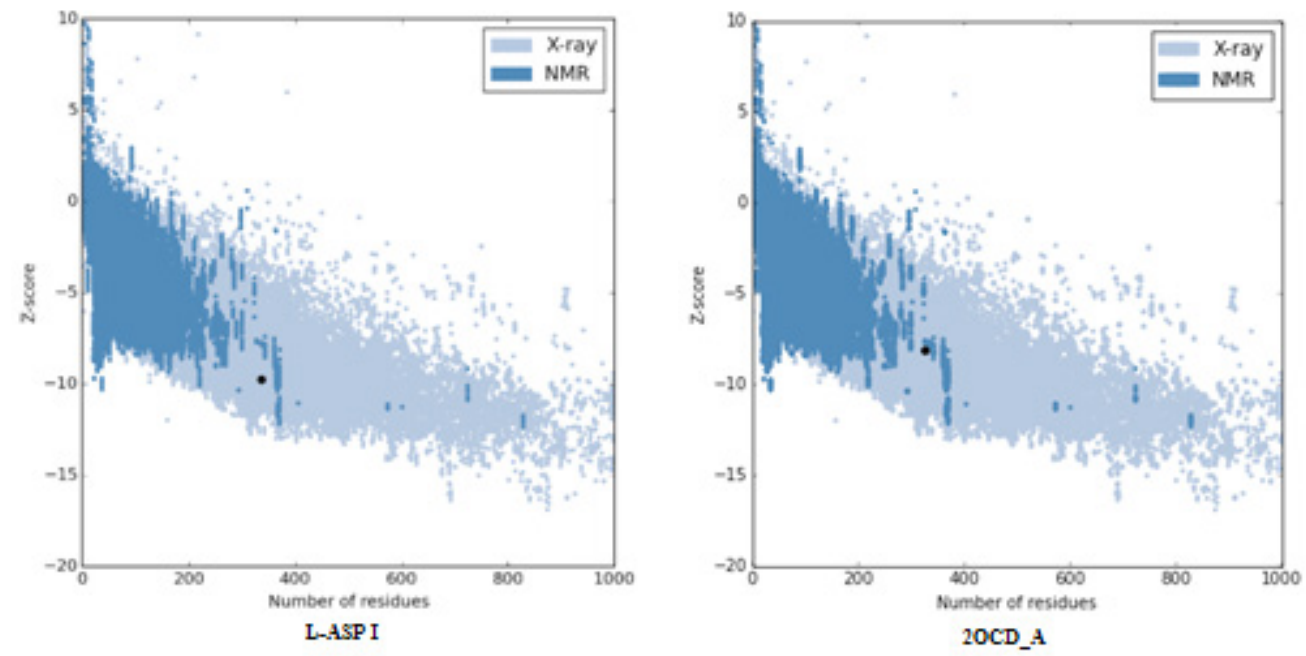

Figure 6. ProSA-web z-scores of all protein chains in PDB determined by X-ray crystallography (light blue) or NMR spectroscopy (dark blue) with respect to their length. The plot shows only chains with less than 1000 residues and a $z$-score $\leq 10$. The $z$-scores of L-ASP I and 2OCD_A are highlighted as large dots. 


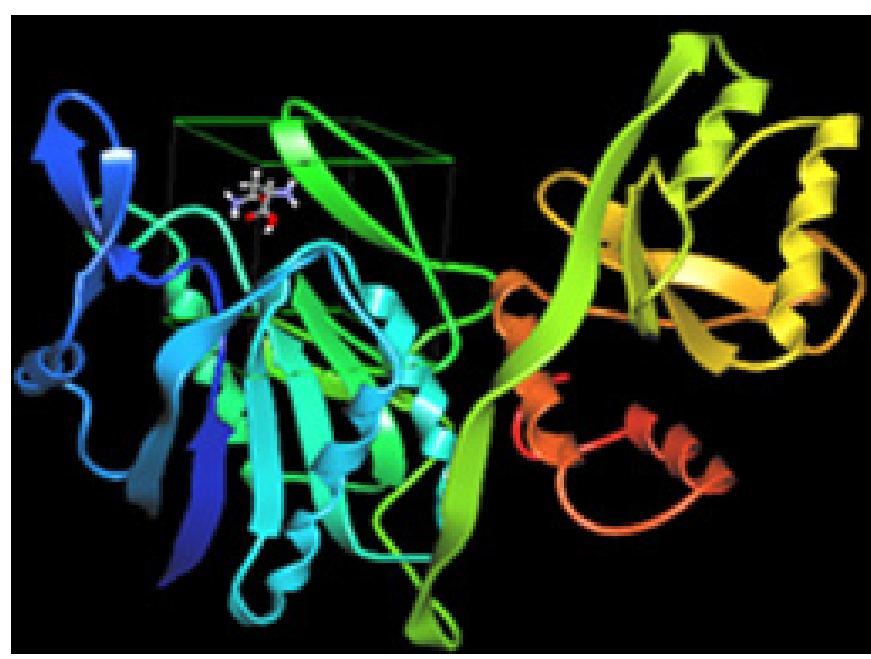

Figure 7. Molecular docking result of L-Asparaginase I and L-asparagine obtained from Argus Lab v.4.0, showing the ligand substrate binding with the enzyme. The ligand substrate is bound to the active site of the larger $\mathrm{N}$-terminal domain of the enzyme.

\section{Protein model validity}

The geometrical and structural consistency of the modeled enzyme was evaluated by different. The $\Phi$ and $\Psi$ distributions of Ramachandran plot analysis using PROCHECK revealed that 93.4\% and 5.6\% amino acid residues are present in the most favored and additionally allowed regions, while $0.7 \%$ and $0.3 \%$ amino acid residues in the generously allowed and disallowed regions. This apparently indicates the geometric fitness of the modeled enzyme (Fig. 5).

The L-ASP I model quality was assessed with the template PDB ID: 2OCD_A (29), based on high sequence identity (85\%). Calculation of RMSD between the modeled enzyme and 2OCD_A was found to be $0.83 \AA$. It determines the best-aligning pair of chains between the template and predicted structure. ERRAT plot shows an overall quality factor of $83.90 \%$, which is indicative of good resolution structure. The $\mathrm{Z}$ score was predicted to be -9.75 in L-ASP I and -8.08 in PDB ID: 2OCD_A (Fig. 6). Protein quality of the structure reveals LG score: 7.129 ( $>4$ : extremely good model) and MaxSub score: 0.568 ( $>0.5$ : very good model) were in the acceptable range of a good model.

The newly generated 3D model of L-ASP I after validation was deposited in Protein Model Database (PMDB). The L-ASP can be accessed from the archive using the Accession No.PM0080443 (https://bioinformatics.cineca.it/PMDB ).

\section{Functional characterization of the predicted structure}

The dimeric form of L-ASP I contain several sites in both the structural domains predicted by Prosite. The residues of each active site from the $\mathrm{N}$-terminal domain show proximity with the C-terminal domain.

The larger N-terminal domain contains two signature motifs for L-ASP I from the residues 8-16 (IAYTGGTIG) and 84-94

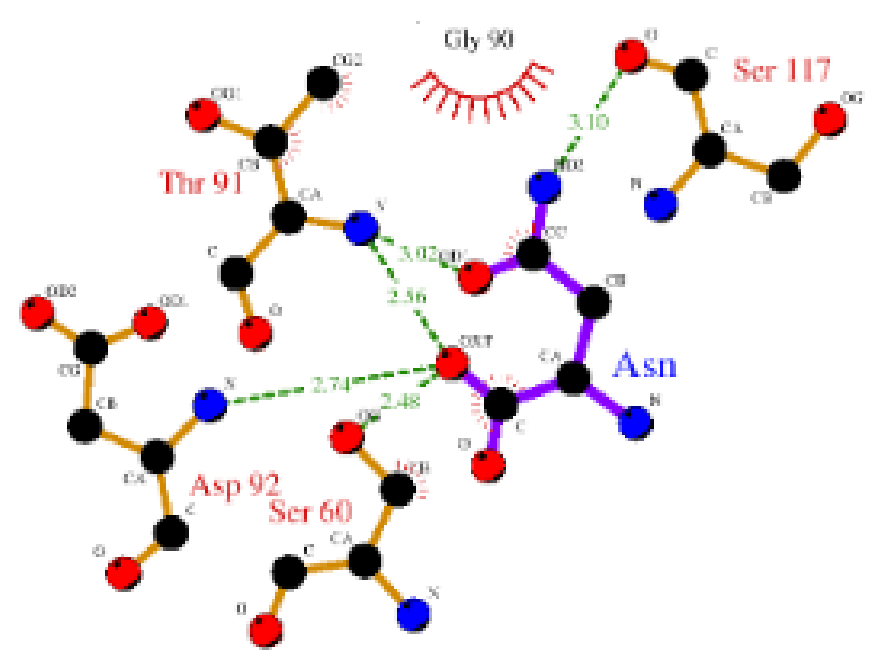

Figure 8. LigPlot showing the docked complex of ligand substrate L-asparagine and enzyme L-Asparaginase. The ligand L-asparagine (Asn) is bound to the enzyme within the sphere of the radius $8 \AA$. The enzyme residues are close to L-Asn (central residue) along the $\mathrm{N}$ and $\mathrm{C}$ direction contribute to short and medium range interaction.

(GFVILHGTDTM) respectively. It also possesses two N-glycosylation sites positioned from the residues 159-162 (NRST) and 192-195 (NITV). The other motifs include Tyrosine sulfation site (residues 42-56, 47-61 and 75-89), Protein kinase C phosphorylation site (residues 2-4, 161-163), Casein kinase II phosphorylation site (residues 37-40, 50-53, 64-67 and 164167) and N-myristoylation site (residues 12-17, 16-21 and 158163).

The smaller C-terminal also contains $\mathrm{N}$-glycosylation site (residues 270-273) NLTQ, Tyrosine sulfation site (residues 313-327), Protein kinase C phosphorylation site (residues 230232 and 262-264), Casein kinase II phosphorylation site (residues 297-300 and 319-322) and N-myristoylation site (residues 282-287, 287-292 and 294-299). The active site residues of the modeled enzyme were predicted to be $14 \mathrm{~T}, 60 \mathrm{~S}, 91 \mathrm{~T}, 92 \mathrm{D}, 117 \mathrm{~S}$, $118 \mathrm{Q}$ and $170 \mathrm{~N}$ by Galaxy server.

Docking of ligand substrate L-asparagine to L-asparaginase I enzyme

When molecular docking runs were performed, the enzyme L-ASP I displayed high binding affinity and significant preference to its substrate L-Asn from its larger $\mathrm{N}$-terminal domain (Fig. 7). The binding energy was recorded to be $-7.45 \mathrm{kcal} / \mathrm{mol}$. The results were confirmed by PatchDock with similar binding efficiency. The calculated global energy was found to be -16.18 $\mathrm{kcal} / \mathrm{mol}$.

The surrounding residues of L-Asn and L-ASP I enzyme are shown within the sphere of the radius $8 \AA$. The enzyme residues T14, T91, S117, Q118, D92 and N170 are close to L-Asn (central residue) along the $\mathrm{N}$ and $\mathrm{C}$ direction contribute to shortrange interactions, while residue $\mathrm{S} 60$ show medium range interaction with the bound L-Asn (Fig. 8) .

The distance between the hydrogen bond lengths were mea- 
Table 2. Molecular interactions between ligand substrate L-Asn and L-ASP I of V. campbellii.

\begin{tabular}{|c|c|c|c|c|}
\hline S.No. & Ligand & Residue and atom & Ligand atom & Bond length $\left(A^{\circ}\right)$ \\
\hline 1 & L-Asn & T14 \& OG1 & $\begin{array}{c}\mathrm{OC} \\
2 \mathrm{HD} 2\end{array}$ & $\begin{array}{l}2.4 \\
2.6\end{array}$ \\
\hline 2 & & S60 \& OG & 0 & 3.5 \\
\hline 3 & & T91 \& OG1 & $\begin{array}{c}\mathrm{OD} 1 \\
1 \mathrm{H}\end{array}$ & $\begin{array}{l}1.7 \\
2.8\end{array}$ \\
\hline 4 & & D92 \& OD1 & $1 \mathrm{H}$ & 2.4 \\
\hline 5 & & S $117 \& 0$ & $2 \mathrm{HD} 2$ & 2.3 \\
\hline 6 & & Q118 \& O & $1 \mathrm{HD} 2$ & 2.3 \\
\hline 7 & & N170 \& ND2 & OD1 & 2.4 \\
\hline
\end{tabular}

sured between ligand substrate L-Asn and the modeled enzyme L-ASP I (Fig. 9). The atom OG1 of T14, T91 base bond takes part in bidentate interactions with OC and 2HD2, OD1 and $1 \mathrm{H}$ of L-Asn. The atom O of S117 and Q118 are involved in a single interaction with 2HD2 and 1HD2 of L-Asn. The ND2 of N170, OD1 of D92 and OG of S60 interact with OD1, 1H and $\mathrm{O}$ of L-Asn, respectively (Table 2). Based on these correlations it was also observed that the enzyme residues T14, S117 and D92 come together in a configurational manner forming a triad, exhibiting a possible role as a catalyst in enzyme-substrate interactions (Fig. 10).

\section{Discussions}

The potential uses of L-ASP as anticancer or anti-leukemic agent for the past few decades have completely revolutionized research in a new direction. These natural bioactive compounds

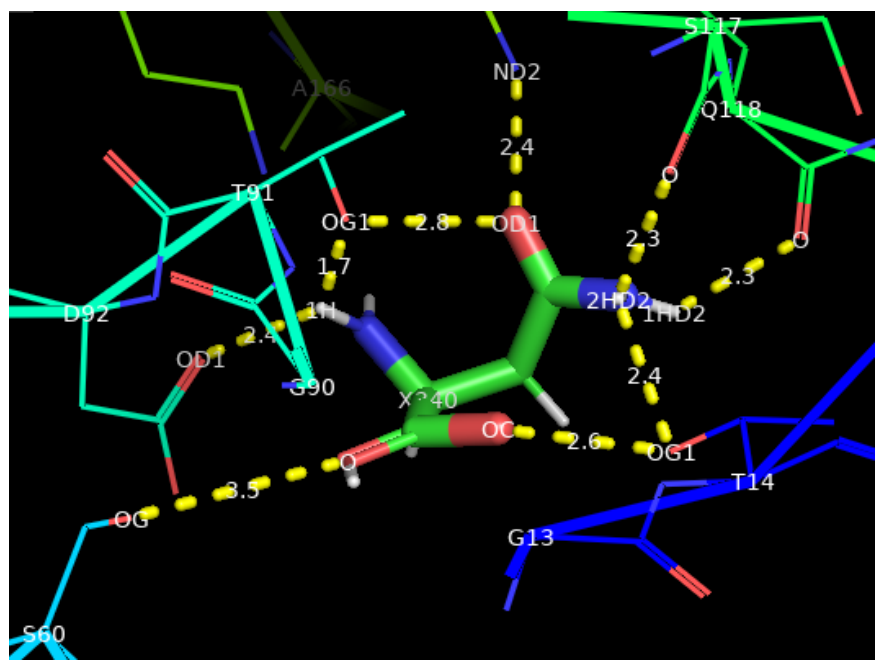

Figure 9. Molecular interactions between L-asparaginase I and ligand substrate L-Asn substrate showing the active site of L-asparaginase I dimer. The side chain carboxyl group of the L-Asn (Central residue) is in close contact with the hydroxyl groups of both candidate nucleophiles, T14 and T91. isolated from a variety of microbes are considered to be safe and less toxic compared to synthetic drugs. In order to study the efficacy of these compounds, it is essential to understand its structure and function. The prediction of ligand binding sites is important in drug discovery to facilitate the optimization process. Most desirable therapeutic agents are usually required in micro quantities but expressing a high degree of purity and specificity.

In the present study, molecular docking results provided detailed structural insights between L-ASP I and ligand substrate L-Asn interactions. The enzyme L-ASP I was predicted to be dimeric and have a molecular weight of $36.9 \mathrm{kDa}$. The molecular weight of enzymes plays an important role in the mechanism of enzyme action. The theoretical isoelectric point demonstrates its acidic nature $(\mathrm{pI}<7)$ and probable role in buffer systems of blood. The GRAVY value is $\leq 0$ indicates hydrophilic

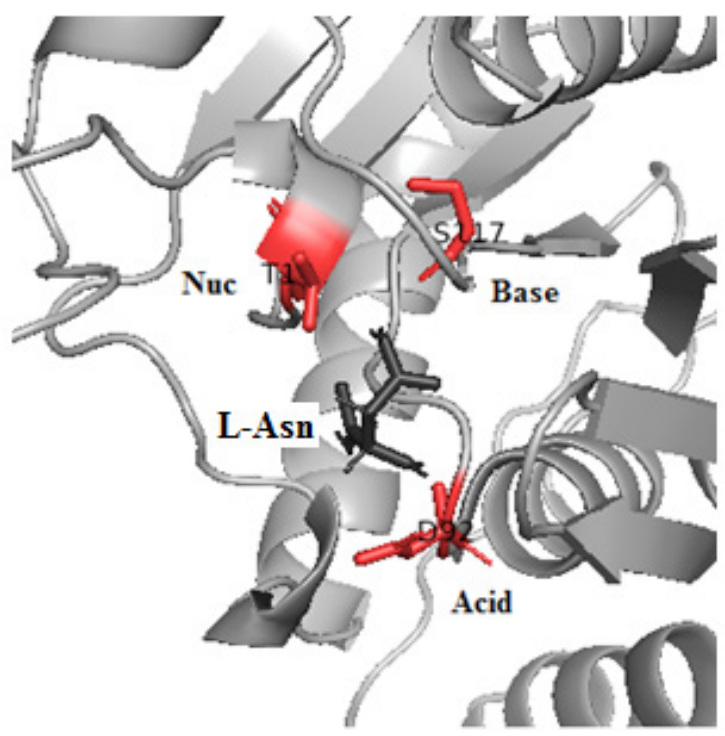

Figure 10. The enzyme L-asparaginase I showing a catalytic triad containing residues (red) in its active site. The triad comprising of residues threonine (nucleophile), serine (base) and aspartic acid (acid). The L-asparagine substrate (black) is bound to the enzyme. 
nature having an affinity to the surrounding water molecules (30). The enzyme predicted to be as non-allergen and close to Apolipoprotein B, a major protein constituent of chylomicrons. Apolipoprotein B-100 functions as a recognition signal for cellular binding (31). However, several adverse effects of L-ASP applications have been reported earlier due to immunogenic reactions (32). In order to alleviate side effects in the treatment process drugs such as Oncaspar and Pegasparase are used in patients whose cancer has not been treated or cannot be treated with asparaginase. These are pegylated asparaginase (poly ethylene glycol linked to asparaginase) which enhances stability, safety and efficacy of the drugs (33).

Interestingly, the predicted 3D structure of L-ASP I has the characteristic features of L-ASP family, designated as Type I cytosolic enzyme. The $\Phi$ and $\Psi$ distributions of Ramachandran plot analysis revealed that $93.4 \%$ and $5.6 \%$ amino acid residues are present in the most favoured and additionally allowed regions, while $0.7 \%$ and $0.3 \%$ amino acid residues in the generously allowed and disallowed regions. This is in conformity with V. cholerae L-ASP I which exhibited $89.2 \%$ and $10.2 \%$ amino acid residues in the most favoured and additionally allowed regions, while $0.4 \%$ and $0.3 \%$ amino acid residues found in the generously allowed and disallowed regions (PDB: 2OCD). Similarly, the Escherichia coli L-ASP (EcA I) showed the presence of $92.2 \%$ residues in the most favourable region, $7.4 \%$ in the allowed region and none in the disallowed region (34). In addition, PROCHECK analysis revealed the main chain and side chain parameters were found to be within the acceptable range indicating the reliability of L-ASP I structure.

The structural convergence includes root mean square deviation (RMSD), low RMSD value between L-ASP I and template PDB ID: 2OCD showed the stability of the predicted structure, while Errat value indicated the good resolution of the structure. ProSA analysis predicted negative interaction energies (-9.75) for most of the residues in L-ASP I, confirming a stable structure. The point of the structure was within a range as demonstrated by X-ray and NMR studies. The model quality and location of the $\mathrm{Z}$-score is in conformity with the template PDB ID: 2OCD.

The modeled enzyme belongs to $\alpha / \beta$ class of proteins characterized by alternating $\alpha$-helices/ $\beta$-sheets running along the backbone of the $\mathrm{N}$-terminal domain. The larger $\mathrm{N}$-terminal domain has an unusual left-handed $\beta-\alpha-\beta$ crossover normally found in a bacterial protein, flavodoxin. This type of arrangement serves as a cavity or cleft for the active site, involved in ligand binding (35). The enzyme is dimeric containing two structural domains with several active sites. The two monomers are held together by a linker amino acid sequence comprising of coils. The coil proteins are long insoluble fibres involved in the extracellular matrix, leading to enzyme-substrate interactions. The larger $\mathrm{N}$-terminal structural domain forms the outer surface of the enzyme while the smaller C-terminal domain is located at the distal region of the domain interface. The present study is in conformity with the crystal structure of Escherichia coli asparaginase II, showing similar structural details (36).
The modeled enzyme showed two signature motifs for L-ASP I activity. It also represented three $\mathrm{N}$-glycosylation sites in the larger and smaller structural domains, where carbohydrates can be linked to proteins through asparagines (N-linked). An asparagine residue can accept an oligosaccharide only if the residue is part of an L-Asn-X-Ser or L-Asn-X-Thr sequence in which ' $\mathrm{X}$ ' can be any residue except proline. Glycosylation enhances the stability of the protein in the blood. Unglycosylated protein has only about $10 \%$ of the bioactivity of the glycosylated form because the protein is rapidly removed from the blood by the kidneys. Thus, potential glycosylation sites can be detected with amino acid sequences. Many proteins, especially those that are present on the surfaces of cells are secreted; acquire carbohydrate units on specific asparagine residues. The addition of sugars makes the proteins more hydrophilic and able to participate in interactions with other proteins (37).

The structure-activity relationship is well demonstrated in the present investigation between the enzyme L-ASP I and ligand substrate L-Asn interactions. A number of distinguishing characteristics are associated with a binding pocket/cleft in L-ASP I. L-Asn access to the binding pocket is facilitated through the $\mathrm{N}$-terminal region of the enzyme. Access is gained by the L-Asn entering through $\alpha 1-\beta 1, \alpha 2-\beta 2$ and $\beta 5-\beta 6$ within the larger structural domain of the enzyme. This is in accordance with the crystal structure of E. coli L-asparaginase II showing the presence of active sites in the larger structural domain of the tetramer (38).

In the present investigation, L-ASP I of $V$. campbellii showed high binding affinity with substrate L-Asn. The binding free energy was found to be $-7.45 \mathrm{kcal} / \mathrm{mol}$. In a similar study, L-asparaginase from Erwinia chrysanthemi_displayed high binding affinity with L-Asn. The binding energy was calculated to be $-52.63 \mathrm{kcal} / \mathrm{mol}$ (39). In fact, low binding free energy confirms L-ASP I and L-Asn complex to be more stable.

The side chains of enzyme residues T14, S60, T91, S117, Q118, D92 and N170 showed a close affinity to the bound L-Asn substrate using ArgusLab v4.0. The residues T14, T91, S117 and S60 are considered to be potential nucleophiles, whereas S60 and S117 serve as a base while D92 is an acid and Q118 is neutral. It is observed from the docked complex that amino acids T14, S117 and D92 come together in a sequential configuration due to the folding of protein residues of the enzyme. These three residues form a catalytic triad (Thr-Ser-Asp) and facilitate binding with the substrate to perform covalent catalysis with the help of T14, which targets the carbonyl carbon of L-Asn and pressurizes the carbonyl oxygen to accept an electron. The T14 is polarized and oriented by S117, which may contribute to the action of T14 as a nucleophile and gets stabilized by D92 within the active site. Though T91 found to be in close contact with L-Asn it is worth mentioning here that OG1 of T91 interacts with $1 \mathrm{H}$ of L-Asn substrate, while OG1 of T14 interacts with OC of L-Asn which is essential for nucleophilic activity. This is in conformity with the catalytic triads commonly found in hydrolase and transferase enzymes (40). Earlier reports of L-ASP crystal structures demonstrated the 
presence of two catalytic triads in each active site. The triad I is composed of a nucleophile (Thr), a base (Tyr) and an acidic moiety (Glu) which functions on the flexibility of the active site loop (41). A similar kind of mechanism may take place when T14 gets activated along with other two residues S117 and D92 leading to the conversion of substrate L-Asn to aspartic acid and ammonia.

Based on the structural evidence, it is therefore derived that T14 is the most potential candidate which acts as a primary nucleophile. The present investigation is in agreement with the modelled crystal structure of the inactive T89V mutant, refined with the aspartate ligand which was covalently bound to T12 by its side chain carboxy group indicating that T12 may function as a primary nucleophile, while this activity may not occur due to the lack of a nucleophilic group on residue T89 (42). In the crystal structure of Escherichia coli L-asparaginase, active sites from $\mathrm{N}$ to $\mathrm{C}$-terminal domains contain diverse subunits. The presence of two threonine residues of the enzyme T12 and T89 may act as primary nucleophiles. However, T89 exhibited as an essential nucleophile, which is fundamental for the enzymatic action (43).

The biological activity of any drug is dependent on the binding between protein and ligand (44). The present investigation has clearly demonstrated a close affinity between the enzyme and its ligand substrate. Hence, L-ASP I of V. campbellii can serve efficiently as anti-leukemic agent in the treatment and control of ALL.

\section{Conclusion}

The predicted structure of L-ASP I of V. campbellii outlined in the current study provides a detailed view of the structure-activity relationship between the enzyme-substrate interactions. The efficacy of L-ASP was mostly attributed to periplasmic L-ASP Type II whilst the current investigation has explicitly demonstrated for the first time that cytosolic L-ASP Type I of $V$. campbellii to possess similar kind of structural and functional activities. Potential residues involved in enzymatic action were identified as T14, S117 and D92 (catalytic triad). The amino acid T14 is identified as a primary nucleophile which is essential for catalytic reaction. Moreover, the scope and applications of asparaginases are well established as anti-leukemic agent for drug targeting and consequent medical applications. Judicious use of these enzymes as biocatalysts will certainly bring promising results in cancer research. . This in silico approach creates new vistas to further explore the active sites of L-ASP I enzyme and to conjugate with poly ethylene glycol (pegylation), which will ensure stability, safety and efficacy of this anti-leukemic agent in the treatment, control and management of ALL.

\section{Acknowledgments}

The author would like to thank the Management, Principal and Head, Department of Zoology, The New College (Autonomous), Affiliated to University of Madras, Chennai-600014, India for their support and encouragement.

\section{Conflict of Interest}

The author declares that there is not conflicts of interest.

\section{Ethical Compliance}

This article does not contain any studies involving human participants or animals performed by the author.

\section{Funding}

This research did not receive any specific grant from funding agencies in the public, commercial, or not-for-profit sectors.

\section{References}

1. Michalska K, Bujacz G, Jaskolski M. Crystal structure of plant asparaginase. J Mol Biol. 2006; 360: 105-116.

2. Deb AC. Fundamentals of Biochemistry. New Central Book Agency (P) Ltd. India; 1994.

3. National Cancer Institute. NCl Dictionary of Cancer terms. Bethesda MD. 18.03d. 2018. https://www.cancer.gov/publications/dictionaries/cancer-terms Accessed on 20 Jan 2019.

4. Konevalova NYu, Buyanova SV. Biochemistry Lecture Course. Ministry of Health of Republic of Belarus Vitebsk State Medical Universite. Vitebsk; 2013. p.163.

5. Peterson RE, Ciegler A . L-asparaginase production by various bacteria. Applied Microbiology. 1969; 17(6):929- 930.

6. DeJong PJ. L-asparaginase production by Streptomyces griseus. Applied \& Environmental Microbiology. 1972; 23 (6):1163-1164.

7. Kafkewitz D, Goodman D. L-asparaginase Production by the Rumen Anaerobe Vibrio succinogenes. Applied \& Environmental Microbiology. 1974; 27(1): 206-209.

8. Sarquis MIdeMoura, Oliveira EMM, Santos AS, da Costa GL. Production of L-asparaginase by filamentous fungi. Memórias do Instituto Oswaldo Cruz. 2004; 99(5): 489-492.

9. Cornea CP, Lupescu I, Vatafu I, Caraiani T, Savoiu VG, Campeanu Gh, Grebenisan I, Negulescu Gh.P, Constantinescu D . Production of L-asparaginase II by Recombinant Escherichia coli cells. Roumanian Biotechnology Letters. 2002; 7(3):717-722.

10. Mashburn LT, Wriston JC. Tumor inhibitory effect of $L$ asparaginase from Escherichia coli. Arch Biochem Biophys. 1964; 105:450-453.

11. Pieters R, Carroll WL. Biology and treatment of acute lymphoblastic leukemia. Pediatr Clin North Am. 2008; 55(1):1-20.[PubMed]

12. Goekbuget N, Baumann A, Beck J, Boos J, Brueggemann M, Diedrich $\mathrm{H}$. PEG-asparaginase in adult acute lymphoblastic leukemia (ALL): efficacy and feasibility analysis with increasing dose levels. Blood. 2008; 112.

13. Savitri, Asthana N, Azmi W. Microbial L-Asparaginase: A potent antitumor enzyme. Indian Journal of Biotechnology. 2003; 2:184194.

14. Swain AL, Jaskolski M, Housset D, Rao JK, Wlodawer A. Crystal structure of Escherichia coli L-asparaginase, an enzyme used in cancer therapy. Proc Natl Acad Sci USA. 1993a; 90(4):pp.1474.

15. Dhevagi P, Poorani E . Isolation and characterization of L-Asparaginase from marine Actinomycetes. Indian Journal of Biotechnology. 2006; 5:514-520.

16. Capizzi RL, Bertino JR and Skeel RT. L-asparaginase: Clinical, biochemical, pharmacological, and immunological studies. Ann Intern Med. 1971; 74: 893-901.

17. Davis BD, Mingioli ES. Mutants of Escherichia coli requiring methionine or vitamin B12. Journal of Bacteriology. 1950; 60(1):17-28.

18. Gallagher MP, Marshall RD, Wilson R. Asparaginase as a drug for treatment of acute lymphoblastic leukaemia. Essays Biochemistry. 1989; 24: 1-40.

19. Lubkowski J, Dauter M, Aghaiypour K, Wlodawer A, Dauter Z. Atomic Resolution Structure of Erwinia chrysanthemi L-Asparagi- 
nase Acta Crystlography D. 2003; 59: 84-92.

20. Dimitrov I, Bangov I, Flower DR, Doytchinova I. AllerTOP v.2. A server for in silico prediction of allergens. J Mol Model. 2014; doi: 10.1007/s00894-014-2278-5. [Epub].

21. Geourjon C, Deleage G. SOPMA: Significant Improvements in Protein Secondary Structure Prediction by Consensus Prediction from Multiple Alignments. Comput Appl Biosci. 1995; 11: 681-684.

22. Kuntal BK, Aparoy P, Reddanna P. EasyModeller: A graphical interface to MODELLER. BMC Res Notes. 2010; doi: 10.1186/17560500-3-226.

23. Lovell S C, Davis I W, Arendall III W B, de Bakker P I W, Word JM, Prisant M G, Richardson JS, Richardson D C. . Structure validation by Calpha geometry: phi,psi and Cbeta deviation. Proteins: Structure, Function \& Genetics. 2003; 50: 437-450.

24. Wallner B., Elofsson A 2003. Protein structure prediction and model quality assessment. Protein Sci. 2003; 112: 1073-1086.

25. Shin W H, Lee G R, Heo L, Lee H, Seok C. Prediction of Protein Structure and Interaction by GALAXY protein modeling programs. Bio Design. 2014; 2 (1): 1-11.

26. Thompson M A. Molecular docking using ArgusLab, an efficient shape-based search algorithm and the AScore scoring function. ACS meeting, Philadelphia, 172, CINF 42, PA. 2004.

27. Wallace, A. C., Laskowski, R. A, Thornton, J. M. LIGPLOT-A program to generate schematic diagrams of protein ligand interactions. Prot. Eng. 1995; 8: 127-134.

28. Schneidman-Duhovny D, Inbar Y, Nussinov R, Wolfson H). Nucl Acids Res. 2005 33(Web Server issue):W363-W367.

29. Nocek B, Wu R, Osipiuk J, Moy S, Kim Y, Joachimiak A. Crystal structure of L-asparaginase I from Vibrio cholerae 01 Biovar eltor str. N16961.Midwest Center for Structural Genomics (MCSG). 2006; doi: 10.2210/pdb2OCD/pdb.

30. Gasteiger E, Hoogland C, Gattiker A, Duvaud S, Wilkins MR, Appel $\mathrm{RD}$, Bairoch A. Protein identification and analysis tools on the ExPASy server. In: Walker JM, editor. The Proteomics Protocols Handbook. Clifton, UK: Humana Press. 2005.

31. Chen SH, Yang CY, Chen PF, Setzer D, Tanimura M, Li WH, Gotto AM $\mathrm{Jr}$, Chan L . The complete CDNA and amino acid sequence of human apolipoprotein B-100. J Biol Chem. 1986; 261:12918-12921.

32. Kwon YM, Chung HS, Moon C, Yockman J, Park YJ, Gitlin SD. LAsparaginase encapsulated intact erythrocytes for treatment of acute lymphoblastic leukemia (ALL). J Control Release. 2009; 139 (3):182-9.

33. National Cancer Institute. NCI Dictionary of Cancer terms. https:// www.cancer.gov/publications/dictionaries/cancer-terms/def/ peg-asparaginase. Accessed on 01 Sep 2019.

34. Yun MK, Nourse A, White SW, Rock CO, Heath RJ. Crystal structure and allosteric regulation of the cytoplasmic Escherichia coli L-asparaginase I. J Mol Biol. 2007; doi:10.1016/j.jmb.2007.03.061.

35. Watenpaugh K D, Sieker L C, Jensen L H. Proc Natl Acad Sci USA. 1973; 70: 3857-3860.

36. Swain AL, Jaskolski M, Housset D, Rao JK, Wlodawer A. Crystal structure of Escherichia coli L-asparaginase, an enzyme used in cancer therapy. Proc Natl Acad Sci USA. 1993b; 90(4): p. 1477.

37. Berg JM, Tymoczko JL, Stryer L. Biochemistry, 7th revised International Edition. W.H. Freeman \& Company. 2012.

38. Swain AL, Jaskolski M, Housset D, Rao JK, Wlodawer A. Crystal structure of Escherichia coli L-asparaginase, an enzyme used in cancer therapy. Proc Natl Acad Sci USA. 1993c; 90(4): p.1477.

39. Reddy ER, Babu RS, Chandrasai PD, Madhuri P. Exploration of the binding modes of L-asparaginase complexed with its amino acid substrates by molecular docking, dynamics and simulation. 3 . Biotech. 2016; 6:105.

40. Stryer L, Berg JM, Tymoczko JL. "9 Catalytic Strategies". Biochemistry, 5th edition: San Francisco: W.H. Freeman. 2002. https://en.wikipedia.org/wiki/Catalytic_triad?oldid=745555039. Accessed $20 \mathrm{Jan}$ 2019.

41. Sanches M, Krauchenco S, Polikarpov I. Structure, Substrate Complexation and Reaction Mechanism of Bacterial Asparaginases. Curr Chem Biol. 2007; 12: 75-86.

42. Palm G, Lubkowski J, Derst C, Schleper S, Roehm KH, Wlodawer A. A covalently bound catalytic intermediate in Escherichia coli asparaginase: Crystal structure of a Thr-89-Val mutant. FEBS Lett. 1996; 390: 211-216.

43. Swain AL, Jaskolski M, Housset D, Rao JK, Wlodawer A. Crystal structure of Escherichia coli L-asparaginase, an enzyme used in cancer therapy. Proc Natl Acad Sci USA. 1993d; 90(4):p. 1474.

44. Derst C, Henseling J, Ro“ hm KH. Engineering the substrate specificity of Escherichia coli asparaginase II. Selective reduction of glutaminase activity by amino acid replacements at position 248 . Protein Sci. 2000; 9:2009-2017. 